\title{
Predictores de identidad profesional en estudiantes de psicología: un estudio de análisis de senderos
}

\section{Predictors of Professional Identity in Psychology Students: a Path Analysis Study}

\author{
David Cuadra-Martínez ${ }^{1}$ \\ Daniel Pérez-Zapata ${ }^{2}$ \\ ${ }^{1,2}$ Universidad de Atacama, Facultad de Humanidades y Edu- \\ cación, Departamento de Psicología, Chile \\ José Sandoval-Díaz ${ }^{3}$ \\ Centro de estudios Nuble y Grupo de Investigación Género, Ciu- \\ dadanía y Equidad (GI 170424/EF), Universidad del Bío Bío, Chile
}

\author{
Jorge Valdivia-Díaz ${ }^{4}$ \\ Universidad de Atacama, Facultad de Humanidades y Educación, \\ Departamento de Educación Física, Chile \\ Carla Palma-Flores ${ }^{5}$ \\ Universidad de Atacama, Facultad de Humanidades y Educación \\ Departamento de Educación Básica, Chile \\ Diego Mora-Dabancens ${ }^{6}$ \\ Universidad de Atacama, Facultad de Humanidades y Edu- \\ cación, Departamento de Psicología, Chile
}

Resumen. El objetivo de este estudio fue determinar la relación entre identidad profesional, identidad estudiantil universitaria, autoeficacia académica, autoeficacia profesional, créditos cursados y dificultad percibida ante la carrera, en estudiantes de psicología tradicionales y no tradicionales. Se utilizó un diseño transversal y un muestreo no probabilístico de 136 estudiantes de psicología de una universidad pública del norte chileno. Se aplicaron cuatro escalas y una encuesta sociodemográfica. Se realizó estadística descriptiva, análisis de correlación de Pearson, análisis de varianza (ANOVA) y análisis de senderos. Se encontraron diferencias significativas en la autoeficacia académica y profesional de estudiantes tradicionales y no tradicionales, y un modelo que predice la identidad profesional a partir de las variables identidad estudiantil universitaria, autoeficacia académica, autoeficacia profesional, créditos cursados y dificultad percibida ante la carrera $\left(R^{2}=.24\right)$. Los resultados se discuten en función de la literatura existente.

Palabras clave. Identidad profesional, identidad estudiantil universitaria, autoeficacia, estudiante de psicología, modelo predictivo.

Abstract. The aim of this study was to determine the relationship between professional identity, university student identity, academic self-efficacy, professional self-efficacy, credits taken and difficulty perceived in the career, in traditional and non-traditional psychology students. We used a cross-sectional design and a non-probabilistic sample of 136 psychology students from a public university in the north of Chile. Four scales and a sociodemographic survey were used. Descriptive statistics, Pearson correlation analysis, analysis of variance (ANOVA) and path analysis were performed. Results showed significant differences in the academic and professional self-efficacy grouped into traditional and non-traditional students and a model that predicts professional identity based on the variables of university student identity, academic self-efficacy, professional self-efficacy, credits taken and perceived difficulty in the career $\left(R^{2}=.24\right)$. Outcomes are discussed according to the existing literature.

Keywords. Professional identity, university student identity, self-efficacy, psychology student, predictive model.

${ }^{1}$ David Cuadra-Martínez. Universidad de Atacama. Facultad de Humanidades y Educación, Departamento de Psicología Avenida Copayapu 485, Copiapó, Chile. E-mail: david.cuadra@uda.cl

${ }^{2}$ Daniel Pérez Zapata. Universidad de Atacama. Facultad de Humanidades y Educación, Departamento de Psicología. Chile. E-mail: DIP827@student.bham.ac.uk

Jjosé Sandoval Díaz. Universidad del Bio-Bio. Departamento de Psicología, Chile. E-mail: jsandoval@ubiobio.cl

${ }^{4}$ Jorge Valdivia Díaz. Universidad de Atacama, Facultad de Humanidades y Educación, Departamento Educación Física, Chile. E-mail: jorge.valdivia@uda.cl

${ }^{5}$ Carla Palma Flores. Universidad de Atacama, Facultad de Humanidades y Educación, Departamento Educación Básica, Chile. E-mail: carla.palma@uda.cl

${ }^{6}$ Diego Mora Dabancens. Universidad de Atacama, Facultad de Humanidades y Educación, Departamento de Psicología, Chile.E-mail: diego.mora@uda.cl 


\section{Introducción}

En el enfoque de formación basado en competencias se propone que el estudiante desarrolle una serie de competencias para dominar una tarea, una mayor confianza en su desempeño y una formación más integral. De esta manera, se espera que, durante el tiempo transcurrido en la universidad, el estudiante no solo desarrolle conocimientos teóricos y técnicos, sino también nuevas habilidades, procedimientos, valores, actitudes y la identificación con el rol profesional (Trede, 2012).

Sin embargo, este enfoque formativo no ha estado exento de problemas. Algunos de estos apuntan a la dificultad para relacionar el conocimiento científico técnico que se enseña en la universidad, con los problemas que los futuros profesionales enfrentarán, así como también el menor valor que el conocimiento profesional tiene en los procesos de formación inicial (Donoso \& Corvalán, 2012). Estas condiciones podrían impactar en la identidad profesional (IP) y estudiantil universitaria, además del sentido de autoeficacia para iniciar la carrera estudiantil y posteriormente ejercer la profesión.

En este contexto, la formación de una IP en educación superior parece ser un factor clave, porque se relaciona con el desempeño profesional (Elman, Illfelder, \& Robiner, 2005) y académico (Montes \& Lerner, 2011). Implica un sentimiento de seguridad y una percepción estable de ser parte de un grupo de profesionales en base a la internalización de estándares, conocimientos, desarrollo de habilidades y actitudes que regulan el desempeño de un rol especializado en la sociedad (Sweitzer, 2008).

La IP se construye socialmente mediante la práctica profesional, las prescripciones de la sociedad del trabajo y las tradiciones formativas (Meschman, Erausquin, \& García, 2014). En la formación inicial, el avance en la profesionalización de los estudiantes trae como consecuencia algún grado de desarrollo de la IP (Kaya, Işik, Şenyuva, \& Kaya, 2017). Sin embargo, este desarrollo parece darse más bien asistemáticamente, porque gran parte del ejercicio de la enseñanza en educación superior se focaliza más en la transmisión de teorías científicas que en abordaje planificado de esta identidad (Cuadra, Castro, \& Juliá, 2018).

De manera específica, esta identidad se construye a partir de la naturaleza de la profesión (Gorman \& Sandefur, 2011), los gremios de profesionales y sus estrategias de negociación sindical, así como el prestigio e incluso el género de la profesión (Barbour \& Lammers, 2015). Para Barbour y Lammers (2015), la IP tiene un fuerte componente grupal, por los roles y funciones que se definen para los profesionales, desde los grupos sociales que demandan atención y aquellos a los que se pertenece.

En línea con lo anterior, para la comprensión de la IP, los estudios actuales la han abordado desde cuatro dimensiones: (a) los estudios que abordan la IP desde el sentido de pertenencia a un grupo de profesionales, (b) aquellos que consideran el apego e involucramiento con la profesión, (c) trabajos que apuntan a comprender cómo las características de las organizaciones definen la IP y (d) los que ponen acento en las creencias acerca de una profesión (Barbour \& Lammers, 2015).

Por otra parte, los estudiantes de educación superior también construyen identidad universitaria. Se entiende por identidad estudiantil universitaria, al grado en que los 
estudiantes conocen y comparten valores, tradiciones, símbolos, aspiraciones, objetivos y metas como estudiantes universitarios (Arteaga, Joya, \& Bastidas, 2014). Se construye desde la experiencia escolar pasada, las relaciones interpersonales y la percepción de la universidad como una unidad (Villanueva et al., 2006). Esta identidad se asocia con las tasas de retención de los estudiantes, la continuidad de estudios, el éxito académico y la motivación académica (González, 2010).

Desde un enfoque de creencias acerca de la profesión (Barbour \& Lammers, 2015), la identificación de los estudiantes con determinado grupo, puede depender de las características sociales, el prestigio y el estatus social de estos grupos (Giddens, 2010), en donde las creencias de autoeficacia son una fuente importante de valoración personal en una dimensión específica: la capacidad para enfrentar tareas, en este caso, aquellas relacionadas con las tareas propias del grupo con el cual se identifica en la universidad y, más tarde, con las que impone el grupo de profesionales al cual pertenecerá.

Así, las creencias de autoeficacia son importantes de desarrollar en el estudiantado, porque afectan el interés y disposición por la tarea, regulan la persistencia, las metas, las elecciones, además de las estrategias cognitivas, metacognitivas y autorreguladoras involucradas en la tarea (Linnenbrink \& Pintrich, 2003). Implican aquellos juicios que una persona hace sobre su capacidad para organizar y ejecutar cursos de acción, necesarios para alcanzar resultados.

Para Bandura (2006) la autoeficacia a nivel específico es más determinante en el comportamiento que la autoeficacia general, porque las personas no son autoeficaces en todo, sino que difieren en las áreas en las que desarrollan su sentido de autoeficacia. En los estudiantes universitarios, la autoeficacia académica y la autoeficacia profesional son dominios específicos necesarios de estudiar, por el sentido que cobran como áreas de funcionamiento en la vida de éstos (Bandura, 2006).

La autoeficacia académica se relaciona con las metas académicas construidas e implica juicios evaluativos sobre la capacidad para lograrlas (Blanco, Ornelas, Aguirre, \& Guedea, 2012). Actualmente, hay evidencia de que estudiantes con alta autoeficacia académica utilizan más estrategias cognitivas para aprender, y autorregulan más su propio aprendizaje (Alegre, 2014). Otros hallazgos permiten relacionar la autoeficacia académica con la toma de decisiones sobre la carrera profesional (Camposeco, 2012).

Por otra parte, la autoeficacia profesional se relaciona con las creencias acerca del desempeño en una tarea profesional. Determina la disposición para resolver problemas profesionales, así como las percepciones de control sobre el entorno laboral; además de la satisfacción con el trabajo (Salanova, Bresó, \& Schaufeli, 2005).

Algunos estudios evidencian que la autoeficacia profesional es menor durante la formación inicial que cuando se ejerce profesionalmente, por ejemplo, en profesores (Penrose, Perry, \& Ball, 2007). En esta misma línea, se ha encontrado que las actividades de enseñanza relacionadas con la práctica del ejercicio profesional, se relacionan con un aumento en la autoeficacia profesional (Velthuis, Fisser, \& Pieters, 2014). 
Respecto de la relación entre las variables anteriormente señaladas, en las teorías evolutivas y sociales se señala que la IP se construye a partir de la forma en que la persona enfrenta una serie de tareas, y la manera en que la profesión se posiciona socialmente. Desde un enfoque evolutivo, se asume que el ser humano, durante su adolescencia, tiene la tarea fundamental de establecer la ocupación que desarrollará en su vida. Por lo que la orientación vocacional cobra importancia durante este período de desarrollo humano (Ruvalcaba, Uribe, \& Gutiérrez, 2011). Una perspectiva más social, destaca el sentimiento de pertenencia a una profesión, desarrollado a partir de la interacción con otros en el contexto de la profesión (Briggs, 2007). Desde las perspectivas anteriores, una mayor madurez profesional, implica una mayor autoeficacia y confianza en las capacidades para abordar las tareas asociadas a la profesión, lo que podría favorecer la construcción de esta identidad (Camposeco, 2012).

Algunas investigaciones actuales han presentado evidencia de lo anterior (Cara \& Riveros, 2016; Flowers \& Banda, 2016). Para ilustrar esta relación, Canrinus, Herms, Beijaard, Buitink y Hofman (2012), al estudiar la formación de la IP en profesores, encontraron que la autoeficacia es un factor predictivo de la IP. En otros estudios se señala que la autoeficacia es un factor importante para la transición de una identidad estudiantil hacia una profesional (Lefroy et al,. 2017). Respecto de esto, Covarrubias (2013) plantea que es en los espacios de socialización en el contexto educativo, donde se va construyendo la IP, en donde la interacción con los pares y profesores parece ser un factor que permite el aprendizaje de los marcos referenciales de la profesión. El estudiante debe ser capaz de identificarse como tal y aprender a ejercer este rol, constituyéndose esto como un factor importante de retención en la universidad (González, 2010).

En la formación profesional de psicólogos, la construcción de una IP es una tarea compleja y a veces difusa para los estudiantes. Para algunos autores, esto se debe al cuestionamiento histórico de la psicología como ciencia, así como a la instalación de un rol social difuso, que incluye distintas funciones e incluso contradicciones a la hora de abordar el comportamiento humano (Covarrubias, 2013). Elman et al. (2005) han encontrado que los psicólogos son más capaces de brindar una atención profesional de calidad cuando construyen una IP, siendo fundamental la internalización de los estándares profesionales; lo cual se logra con variados métodos formativos.

Rønnestad y Skovholt (2003) describen esta construcción como un proceso en etapas, que se da en la medida que los estudiantes avanzan en sus niveles formativos. A partir de esta perspectiva, el tiempo transcurrido en la universidad podría ser un factor que impacta en el desarrollo de la IP. Según estos autores, en este proceso influirían a) el conocimiento, b) crecimiento personal, c) experiencia, d) percepciones sobre la profesión e) las experiencias de logro, f) los costos y g) las relaciones interpersonales del estudiante en formación. Siendo este último factor, uno de los más determinantes. No obstante, esta propuesta no deja claro si en todos los niveles formativos de la formación inicial, la IP se desarrolla con la misma intensidad y características.

Por otra parte, la formación de una IP podría verse afectada por el acceso cada vez mayor a las universidades de aquellos estudiantes denominados no tradicionales (Tilley, 
2014). Un estudiante no tradicional es aquel que además de estudiar, presenta una mayor responsabilidad familiar y/o laboral, un atraso en el acceso a la educación superior, que pertenece a un grupo étnico, o presenta una discapacidad. Este tipo de estudiantes presentan una tasa de abandono más alta al interior de los estudiantes universitarios (Sánchez \& Andreu, 2017).

A pesar de las mayores dificultades que los estudiantes no tradicionales encuentran en el contexto de la educación superior, éstos presentarían mayor motivación intrínseca y persistencia (Scott \& Lewis, 2012), un comportamiento más orientado al aprendizaje (Hoyert \& O’Dell, 2009) y estilos de afrontamiento más adaptativos frente al estrés. Precisamente la dificultad percibida de una tarea, en este caso el cursar una carrera profesional, ha sido considerada como una variable importante e influyente en la IP y la motivación. Las características de una tarea reportan información de la congruencia, dificultad e importancia de ésta para la IP. Por ejemplo, tareas percibidas como demasiado fáciles o como imposibles de realizar, representarían un sinsentido para la IP (Oyserman, 2015).

Como disciplina y profesión, la psicología atrae a un número importante de estudiantes, y muchos de éstos clasifican como no tradicionales (Zinkiewicz \& Trapp, 2004). Taylor y House (2010) han encontrado diferencias en la construcción de la identidad, las expectativas y motivaciones entre estudiantes de psicología tradicionales y no tradicionales. Lo estudiantes maduros o de mayor edad, tienden a reportar un mayor cambio en su identidad personal al progresar en la carrera. También se ha encontrado diferencias en la motivación intrínseca y extrínseca, siendo más frecuente que los estudiantes no tradicionales reporten motivaciones tanto intrínsecas como extrínsecas por estudiar psicología. Las preocupaciones principales de los estudiantes no tradicionales se centran en lo académico y financiero, mientras que los tradicionales se centran más en problemas sociales.

Por lo tanto, el objetivo de este trabajo es determinar la relación entre identidad profesional, identidad estudiantil universitaria, autoeficacia académica, autoeficacia profesional, créditos cursados y dificultad percibida en la carrera, en estudiantes de psicología tradicionales y no tradicionales. A nivel específico se busca: (a) describir la forma en que se relacionan estas variables; (b) determinar los niveles de identidad profesional, identidad estudiantil universitaria, autoeficacia profesional y académica en función de las variables tipo de estudiante (tradicional - no tradicional) y sociodemográficas (edad, sexo, ingreso económico); y (c) proponer un modelo que explique la identidad profesional de estudiantes de psicología, en función de las variables autoeficacia académica, identidad estudiantil universitaria, autoeficacia profesional, créditos cursados en la carrera y percepción de dificultad ante la misma.

Para lo anterior, se trabajó con base en las siguientes hipótesis:

H1: Se presenta una relación entre las variables identidad profesional, identidad estudiantil universitaria, autoeficacia académica, autoeficacia profesional, créditos cursados y dificultad percibida en la carrera en estudiantes de psicología tradicionales y no tradicionales.

H2: Hay diferencias significativas en los niveles de identidad profesional, identidad estudiantil universitaria, autoeficacia profesional y académica en función de las variables 
tipo de estudiante (tradicional - no tradicional); y sociodemográficas (edad, sexo, ingreso económico).

H3: Las variables autoeficacia académica, identidad estudiantil universitaria, autoeficacia profesional, créditos cursados y la dificultad percibida en la carrera, son predictoras de la identidad profesional.

\section{Método}

Corresponde a un estudio cuasi experimental, de tipo correlacional, dado que en este trabajo interesa establecer relaciones entre variables; y mediante path analysis explicar esta relación (Díaz, 2016).

\section{Participantes}

Se utilizó un diseño de carácter transversal con un muestreo no probabilístico. Participaron 136 estudiantes de la carrera de psicología de una universidad pública del norte de Chile (primer año $n=41.9 \%$; segundo año $n=25.7 \%$; tercer año $n=19.9 \%$; cuarto año $n$ $=12.5 \%$. El menor número muestral en los últimos años se debe a la reprobación de asignaturas de los estudiantes durante la carrera profesional). La edad promedio fue de 21 años $(D E=2.7), 67.6 \%$ fueron mujeres y $32.4 \%$ hombres, mientras que el $29.4 \%$ fueron estudiantes no tradicionales y el $70.6 \%$ estudiantes tradicionales.

\section{Instrumentos}

Se utilizaron los siguientes instrumentos para medir las variables del estudio:

Encuesta sociodemográfica. Esta encuesta midió las variables sexo, edad, ingreso económico, tipo de estudiante y grado de dificultad percibida ante carrera. Para la tipificación de estudiante tradicional o no tradicional (Sánchez \& Andreau, 2017), se consideró como estudiante no tradicional a aquel que presentó cualquiera de las siguientes condiciones consultadas en este instrumento: (a) trabajar 15 o más horas a la semana, (b) tener 24 o más años de edad (c) mantener una relación de convivencia o encontrarse casado; (d) pertenecer a un grupo étnico, y (e) tener una persona bajo su cuidado. En esta misma encuesta se les preguntó a los estudiantes por el grado de dificultad percibida ante la carrera: a) ¿Qué tan difícil es la carrera que cursas actualmente? (a) muy fácil; b) levemente fácil, c) ni fácil ni difícil, d) levemente difícil, e) muy difícil.

Escala de Identidad Profesional (Adams, Hean, Sturgis, \& Clark, 2006). Corresponde a una escala de autoreporte unidimensional que mide la fuerza de la IP en estudiantes universitarios, a partir de 9 ítems en alternativa de respuesta Likert de 1 (nada de acuerdo) a 5 (muy de acuerdo). En estudios previos, el instrumento ha presentado una consistencia interna aceptable $(\alpha=.79)$. La versión utilizada en esta investigación corresponde a la adaptación al castellano realizada por Negrillo (2015). En nuestro estudio el instrumento presentó una confiablidad aceptable $(\alpha=.66)$.

Escala de identidad estudiantil universitaria (Cabral et al., 2006). Mide el grado de identidad de los estudiantes universitarios hacia su institución. Es una escala unidimensional que cuenta de 20 reactivos con alternativas de respuesta que van de 1 (muy en desacuerdo) a cinco 5 (muy de acuerdo). En este estudio, el instrumento arrojó un $\alpha$ de .78. 
Cuestionario de autoeficacia profesional (AU) (Maffei, Spontón, Spontón, Castellano, \& Medrano, 2010). Es un instrumento unifactorial de 10 ítems que, miden las creencias que se poseen acerca de las capacidades para ejecutar exitosamente actividades asociadas a la profesión. Las alternativas de respuesta van de 0 (nunca o ninguna vez) a 6 (siempre o todos los días). Como parte de las instrucciones, se les solicitó a los estudiantes responder pensando en su futuro profesional, para que las respuestas fueran asociadas al futuro trabajo profesional y no a otro posible oficio actualmente desarrollado. En este estudio el instrumento presentó una confiabilidad alta $(\alpha=.85)$.

Cuestionario sobre autoeficacia académica general (Torre Puente, 2006). Corresponde a un cuestionario de autoreporte con el que se mide la autoeficacia académica general. Presenta una estructura unifactorial y se compone de 9 ítems con alternativa de respuesta tipo Likert en una escala de 1 (en total desacuerdo) a 5 (totalmente de acuerdo). En este estudio el instrumento presentó una confiabilidad alta $(\alpha=.92)$.

Finalmente, se obtuvieron los créditos cursados de los estudiantes (un indicador de las asignaturas cursadas en la carrera), lo que permitió determinar el tiempo transcurrido en la universidad del estudiante de psicología. Los créditos cursados se han utilizado tanto como medida de rendimiento académico (Barahona, 2014), como de tiempo transcurrido en la universidad (Arias \& Pineda, 2011). En esta investigación optamos por utilizarlos como medida del avance académico, con el fin de observar si el tiempo trascurrido en la universidad podía incidir en la identidad profesional.

\section{Procedimentos}

Se contactó a los estudiantes en el aula de clases, a quienes se les explicó de manera general los fines de la investigación. Cada uno de ellos leyó y firmó, al inicio del instrumento, un consentimiento informado de confidencialidad y anonimato. La aplicación de los instrumentos tuvo una duración aproximada de 25 minutos. Los datos se analizaron mediante el programa IBM SPSS 20.0.

Para el análisis de datos se descartaron aquellos puntajes promedios en donde los puntajes z fueran igual o mayor a -2.5 y menor o igual que -2.5 , de acuerdo con la sugerencia de Pérez, Medrano y Sánchez (2013). Luego, se realizó análisis de confiabilidad y correlaciones de Pearson para las variables identidad profesional, identidad estudiantil universitaria, autoeficacia académica, autoeficacia profesional, créditos cursados y dificultad percibida en la carrera. En tercer lugar, se aplicó análisis de varianza (ANOVA) para determinar la existencia de diferencias entre las variables, según tipo de estudiantes y variables sociodemográficas. Finalmente, se realizó análisis de senderos (path analysis) para generar un modelo que permitiera explicar la IP en base a las variables autoeficacia académica, autoeficacia profesional, identidad estudiantil universitaria, créditos cursados y dificultad percibida en la carrea. Para esto último, se utilizó el programa AMOS 21.0 y se trabajó con el método de Máxima Verosimilitud. Se especificó el modelo calculando los grados de libertad, se estimaron los valores de los parámetros especificados a partir de las varianzas y covarianzas muestrales y se evaluó el ajuste del modelo. 


\section{Resultados}

\section{Correlaciones}

Mediante el coeficiente de correlación lineal de Pearson, se relacionó autoeficacia profesional, autoeficacia académica, identidad profesional, identidad estudiantil universitaria, créditos cursados y nivel de dificultad percibida ante la carrera (tabla 1). Entre las 4 primeras variables se encontró una relación positiva y estadísticamente significativa. Las asociaciones más fuertes se presentan entre autoeficacia profesional y autoeficacia académica; y entre identidad profesional e identidad estudiantil universitaria. Autoeficacia académica también correlaciona positivamente con identidad estudiantil universitaria y con identidad profesional. Además, la autoeficacia profesional correlaciona positivamente con identidad estudiantil universitaria y con identidad profesional.

La variable créditos cursados correlaciona negativa y significativamente con las variables identidad profesional, identidad estudiantil universitaria y dificultad percibida en la carrera. La variable dificultad percibida ante la carrera, correlaciona negativa y significativamente con autoeficacia académica. Lo anterior implica que en la medida que los estudiantes avanzan en su carrera profesional, disminuye su identidad profesional y universitaria; además de la percepción de dificultad de la carrera. También que, en la medida que aumenta la percepción de la dificultad ante la carrera, disminuye la autoeficacia académica.

Niveles de autoeficacia profesional, autoeficacia académica, identidad profesional y estudiantil universitaria

Se determinaron los niveles de autoeficacia profesional y académica, e identidad profesional y estudiantil universitaria, en función de las variables tipo de estudiante (tradicional - no tradicional) y sociodemográficas. Calculando mediante análisis de varianza de un factor (ANOVA), la existencia de diferencias significativas de media.

Autoeficacia profesional. Los estudiantes no tradicionales presentan una media de 44.38 en autoeficacia profesional, mientras que los estudiantes tradicionales presentan una media de 42.64, encontrándose diferencias estadísticamente significativas $(p=.05)$.

Tabla 1

Correlación entre las variables del estudio

\begin{tabular}{lcccccc}
\hline & 1 & 2 & 3 & 4 & 5 & 6 \\
\hline 1. Autoeficacia Profesional & 1 & $.56^{* *}$ & $.43^{* *}$ & $.30^{* *}$ & -.11 & -.05 \\
2. Autoeficacia académica & & 1 & $.41^{* *}$ & $.29^{* *}$ & -.09 & $-.24^{* *}$ \\
3. Identidad universitaria & & & 1 & $.44^{* *}$ & $-.38^{* *}$ & -.01 \\
4. Identidad profesional & & & & 1 & $-.20^{*}$ & .10 \\
5. Créditos cursados & & & & 1 & $-.21^{*}$ \\
6. Dificultad percibida en la carrera & & & & & & 1 \\
\hline$* p<.05 . * * p .01$ & & & & &
\end{tabular}


A nivel específico, quienes trabajan 15 o más horas a la semana presentan una mayor autoeficacia profesional que quienes trabajan menos horas o no trabajan. También quienes son solteros presentan mayor autoeficacia profesional que quienes mantienen una relación de convivencia. Las demás variables no presentan diferencias significativas en las medias de autoeficacia profesional (tabla 2).

Autoeficacia académica. Se observan diferencias de medias estadísticamente significativas en la autoeficacia académica de estudiantes tradicionales y no tradicionales, según la variable estado civil. Quienes son solteros poseen una mayor autoeficacia académica que quienes mantienen una relación de pareja de convivencia. Para las otras variables medidas no se encuentran diferencias de media significativas (tabla 3).

Tabla 2

Autoeficacia profesional según tipo de estudiante

\begin{tabular}{|c|c|c|c|c|c|c|c|c|c|}
\hline \multirow{2}{*}{\multicolumn{2}{|c|}{ Autoeficacia Profesional }} & \multirow[b]{2}{*}{$n$} & \multirow[b]{2}{*}{$X$} & \multirow[b]{2}{*}{$\mathrm{EE}$} & \multicolumn{2}{|c|}{ Intervalo de confianza } & \multirow[b]{2}{*}{ F } & \multirow[b]{2}{*}{$a$} & \multirow[b]{2}{*}{$n^{2}$} \\
\hline & & & & & LI & LS & & & \\
\hline \multirow{3}{*}{$\begin{array}{l}\text { Tipo de } \\
\text { estudiante }\end{array}$} & & & & & & & 3.88 & $.05^{*}$ & .03 \\
\hline & Tradicional & 75 & 42.64 & 0.54 & 41.55 & 43.72 & & & \\
\hline & No tradicional & 62 & 44.38 & 0.71 & 42.95 & 45.81 & & & \\
\hline \multirow{3}{*}{$\begin{array}{c}\text { Trabaja } \geq \\
15 \mathrm{Hrs}\end{array}$} & & & & & & & 4.85 & $.05^{*}$ & .03 \\
\hline & Sí & 25 & 45.48 & 0.88 & 43.65 & 47.30 & & & \\
\hline & No & 112 & 42.97 & 0.49 & 41.98 & 43.96 & & & \\
\hline \multirow{3}{*}{$\begin{array}{c}\text { Trabaja } \geq \\
24 \text { años }\end{array}$} & & & & & & & 1.42 & .23 & \\
\hline & Sí & 17 & 44.82 & 1.12 & 42.44 & 47.20 & & & \\
\hline & No & 118 & 43.21 & 0.48 & 42.25 & 44.17 & & & \\
\hline \multirow{3}{*}{$\begin{array}{l}\text { Pueblo } \\
\text { Originario }\end{array}$} & & & & & & & 0.07 & .78 & \\
\hline & Sí & 21 & 43.66 & 1.43 & 40.66 & 46.67 & & & \\
\hline & No & 114 & 43.32 & 0.46 & 42.40 & 44.24 & & & \\
\hline \multirow{3}{*}{$\begin{array}{l}\text { Personas } \\
\text { a cargo }\end{array}$} & & & & & & & 1.51 & .22 & \\
\hline & Sí & 22 & 44.68 & 1.00 & 42.58 & 46.78 & & & \\
\hline & No & 115 & 43.19 & 0.49 & 42.21 & 44.16 & & & \\
\hline \multirow{3}{*}{$\begin{array}{l}\text { Estado } \\
\text { civil }\end{array}$} & & & & & & & 5.87 & $.01 * *$ & .04 \\
\hline & Soltero & 130 & 43.67 & 0.43 & 42.81 & 44.54 & & & \\
\hline & Conviviente & 7 & 38.85 & 2.89 & 31.76 & 45.94 & & & \\
\hline
\end{tabular}


Tabla 3

\begin{tabular}{|c|c|c|c|c|c|c|c|c|}
\hline \multicolumn{2}{|c|}{ Autoeficacia Académica } & \multirow[b]{2}{*}{$X$} & \multirow[b]{2}{*}{ EE } & \multicolumn{2}{|c|}{ Intervalo de confianza } & \multirow[b]{2}{*}{$F$} & \multirow[b]{2}{*}{$a$} & \multirow[b]{2}{*}{$\eta^{2}$} \\
\hline & & & & LI & LS & & & \\
\hline & Tradicional & 35.74 & .46 & 34.81 & 36.67 & & & \\
\hline & No tradicional & 35.81 & .68 & 34.45 & 37.18 & & & \\
\hline \multirow{3}{*}{$\begin{array}{c}\text { Trabaja } \geq \\
15 \mathrm{Hrs}\end{array}$} & & & & & & .04 & .82 & \\
\hline & Sí & 35.96 & 1.06 & 33.76 & 38.15 & & & \\
\hline & No & 35.73 & 0.42 & 34.88 & 36.58 & & & \\
\hline \multirow{3}{*}{$\begin{array}{c}\text { Trabaja } \geq \\
24 \text { años }\end{array}$} & & & & & & .37 & .54 & \\
\hline & Sí & 36.47 & 1.33 & 33.63 & 39.31 & & & \\
\hline & No & 35.73 & 0.42 & 34.89 & 36.56 & & & \\
\hline \multirow{3}{*}{$\begin{array}{c}\text { Pueblo } \\
\text { Originario }\end{array}$} & & & & & & 2.45 & .19 & \\
\hline & Sí & 34.33 & 1.21 & 31.80 & 36.86 & & & \\
\hline & No & 36.06 & 0.42 & 35.22 & 36.89 & & & \\
\hline \multirow{3}{*}{$\begin{array}{l}\text { Personas } \\
\text { a cargo }\end{array}$} & & & & & & 3.19 & .08 & \\
\hline & Sí & 37.38 & 1.30 & 33.82 & 39.26 & & & \\
\hline & No & 35.48 & 0.44 & 34.48 & 36.23 & & & \\
\hline \multirow{3}{*}{$\begin{array}{c}\text { Estado } \\
\text { civil }\end{array}$} & & & & & & 3.93 & $.04 *$ & .03 \\
\hline & Soltero/a & 35.96 & 0.40 & 35.16 & 36.76 & & & \\
\hline & Conviviente & 32.42 & 1.71 & 28.06 & 36.79 & & & \\
\hline
\end{tabular}

Identidad profesional y estudiantil universitaria. No se observan diferencias de media significativas (ANOVA), en función del tipo de estudiante, en ninguna de las variables consideradas para la tipificación de estudiante tradicional o no tradicional, ni en las variables sociodemográficas.

Análisis de Senderos. Se construyó un modelo que aporta en la explicación de la IP a partir de las variables autoeficacia académica, autoeficacia profesional, identidad estudiantil universitaria, créditos cursados y dificultad percibida en la carrera profesional (Figura 1). El modelo presenta adecuados índices de ajuste que se especifican en la tabla 4.

En esta representación gráfica se observa que, autoeficacia académica presenta un efecto directo en la IP $(\beta=.19, p<.05)$. Así, altos niveles de autoeficacia académica están 


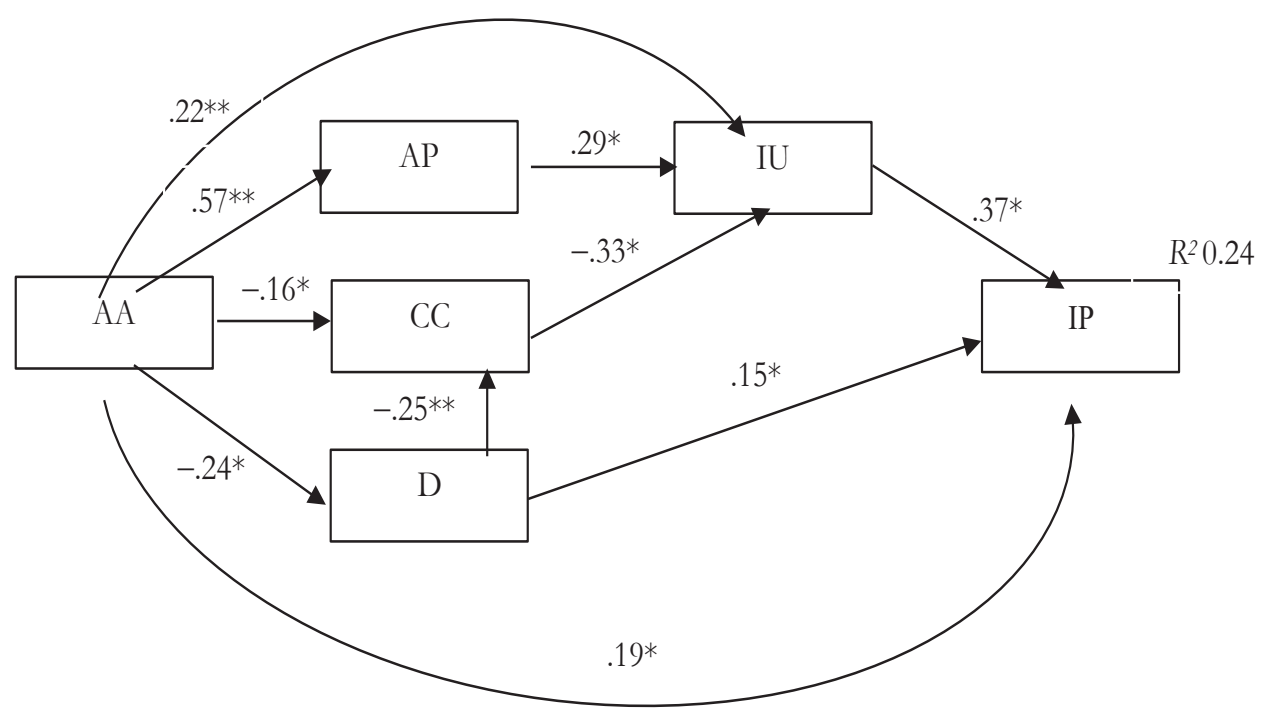

Figura 1. Modelo predictivo de identidad profesional según coeficientes Path. AA= autoeficacia académica; $\mathrm{AP}=$ autoeficacia profesional; $\mathrm{CC}=$ créditos cursados; $\mathrm{D}=$ dificultad percibida en la carrera; $\mathrm{IU}=$ identidad estudiantil universitaria; $\mathrm{IP}=$ identidad profesional. $* p<.05 .{ }^{* *} p<.01$

Tabla 4

Índices de ajustes del modelo

\begin{tabular}{ccc}
\hline Índices de ajustes & $\begin{array}{c}\text { Criterios de referencia } \\
\text { (Bentler, 1995; Byrne, 2001) }\end{array}$ & Modelo \\
\hline$d f$ & $>0$ & 4 \\
Nivel de probabilidad & - & .80 \\
$X^{2}$ & $>.05$ & 1.61 \\
CFI & CFI $\geq .95$ & 1 \\
NFI & $\geq .95$ & .99 \\
RMSEA & $<.05$ & .00 \\
TLI & $\geq .95$ & 1.08 \\
IFI & Cercano a la unidad o superior & 1.01 \\
RFI & Cercano a la unidad o superior & .95 \\
\hline
\end{tabular}

asociados a altos niveles de IP. La identidad estudiantil universitaria $(\beta=.37, p<.01)$ y dificultad percibida en la carrera $(\beta=.15, p<.05)$, también tienen un efecto importante y directo sobre la IP: cuanto mayor sea la identidad estudiantil universitaria y el grado percibido de dificultad en la carrera, mayor será la probabilidad de desarrollar IP.

En cuanto a los efectos indirectos sobre la IP, se observa que la autoeficacia académica presenta una relación positiva y significativa con autoeficacia profesional $(\beta=.57, p<.01)$, la 
cual se asocia a identidad estudiantil universitaria $(\beta=.29 p<.01)$ y esta última con la IP. De esta forma, en la medida que aumentan la autoeficacia académica, autoeficacia profesional e identidad estudiantil universitaria, hay una mayor probabilidad de que aumente la IP.

La autoeficacia académica también se relaciona negativa y significativamente con los créditos cursados en la carrera $(\beta=-.16, p<.05)$, y éstos negativa y significativamente con la identidad estudiantil universitaria $(\beta=-.33, p<.01)$, teniendo esta última un efecto directo sobre IP. Así, un aumento en la autoeficacia académica implica un efecto negativo en los créditos cursados, lo que aumenta la identidad estudiantil universitaria, implicando un mayor desarrollo de la IP.

Finalmente, autoeficacia académica se relaciona negativa y significativamente con el grado de dificultad percibido en la carrera $(\beta=-.24 p<.01)$, y esta última de manera directa con IP. Un aumento de la autoeficacia académica implica una menor percepción de dificultad en la carrera profesional, lo cual podría disminuir el desarrollo de la IP.

Las variables créditos cursados y autoeficacia profesional también tienen un efecto indirecto sobre la IP. En la medida que aumentan los créditos cursados, disminuye la identidad estudiantil universitaria $(\beta=-.33 p<.01)$, lo que repercute en una menor IP. En la medida que aumenta la autoeficacia profesional, aumenta la identidad estudiantil universitaria $(\beta=.29 p<.01)$, lo que repercute en un mayor desarrollo de la IP. Por último, mediante el modelo obtenido se explica el $24 \%$ de la varianza de la IP $\left(R^{2}=.24\right)$.

\section{Discusión}

El objetivo de este trabajo fue determinar la relación entre identidad profesional, identidad estudiantil universitaria, autoeficacia académica, autoeficacia profesional, créditos cursados y dificultad percibida en la carrera, en estudiantes de psicología tradicionales y no tradicionales. Se encontró que estas variables se encuentran relacionadas, resultado que es consistente con la hipótesis 1 de este trabajo. A continuación, se discute en detalle los resultados obtenidos.

A nivel específico, se buscó determinar los niveles de las variables medidas, en función del tipo de estudiante (tradicional - no tradicional). Se propuso como hipótesis la existencia de diferencias significativas en estos niveles, sin embargo, esto se observó sólo en algunas variables. Los resultados de esta investigación muestran algunas variaciones en el desarrollo de la autoeficacia académica y profesional, según el tipo de estudiante. Los estudiantes no tradicionales que trabajan 15 o más horas tienden a desarrollar una mayor autoeficacia profesional que los estudiantes tradicionales. Estos resultados son indicativos de que los estudiantes no tradicionales constituyen un grupo que no solo presenta mayor motivación intrínseca, persistencia (Scott \& Lewis, 2012) y un comportamiento más orientado al aprendizaje (Hoyert \& O’Dell, 2009).

Una posible explicación a esto es que los estudiantes que combinan estudios y trabajo, a partir de esta experiencia laboral desarrollan una mayor disposición para resolver problemas en el trabajo y un sentimiento de mayor control en ese contexto (Salanova et al., 2005). Experiencias que constituyen un aprendizaje para más tarde funcionar en el campo profesional. Estas experiencias laborales, aunque no representan una práctica del ejercicio 
profesional, podrían implicar una forma de entrenamiento de las competencias genéricas asociadas al mundo del trabajo (Velthuis et al., 2014). Lo anterior advierte de la necesidad de que la formación inicial, sobre todo desde un enfoque por competencias, considere experiencias formativas en todos los niveles que, combinen la teoría con la práctica y sobre todo en contextos reales de enseñanza.

Por otra parte, cuando se contrasta la autoeficacia profesional en función de si el estudiante mantiene una relación de convivencia en pareja, los estudiantes no tradicionales que conviven son quienes presentan un menor desarrollo de la autoeficacia profesional, situación que también se da con la autoeficacia académica. Algunas investigaciones han relacionado el aumento de la responsabilidad en los estudiantes que ingresan a la universidad, con episodios de ansiedad y estrés (Martínez-Otero, 2014).

Dado que el estudio en educación superior con frecuencia implica un compromiso pleno, es probable que la mayor responsabilización, en este caso el combinar estudios con una relación de convivencia, no solo sea un posible gatillante de ansiedad, sino que también, una limitante para desarrollar un sentimiento de seguridad respecto del logro académico y profesional.

Olukemi y Awotedu (2014), en un estudio con mujeres universitarias casadas, encontró que uno de los mayores desafíos que enfrentan como estudiantes es contar con el tiempo y espacio adecuado para estudiar, y muchas veces las responsabilidades asociadas a una relación de convivencia o conyugal, son consideradas más importantes que las escolares. La combinación de roles familiares y estudiantiles puede ser una fuente importante de estrés que, conlleva a un sentimiento de menor eficacia para poder lograr cumplir con estas responsabilidades (Egan, 2004).

En cuanto a la IP e identidad estudiantil universitaria, no se encontraron diferencias entre los estudiantes tradicionales y no tradicionales. Estos resultados contrastan con los propuestos por Taylor y House (2010), quienes encontraron que los estudiantes de psicología no tradicionales más maduros, reportan mayores cambios en su identidad al avanzar en la carrera profesional. Esto podría deberse a las características del proceso formativo en la carrera, por ejemplo, las tutorías y asesoramiento psicológico; sobre todo para los estudiantes no tradicionales, por ser quienes probablemente más lo requieren. Desde esto, parece pertinente que futuras investigaciones permitan aportar en comprensión, sobre cómo es que las universidades están formando a este tipo de estudiantes, en este caso, en las carreras de psicología, por ser una de las que atrae a un número importante de estudiantes no tradicionales (Zinkiewicz \& Trapp, 2004).

Sin embargo, la IP e identidad estudiantil universitaria muestran una relación significativa y negativa con los créditos cursados. Estos resultados podrían atribuirse a la dificultad de la sociedad actual para promover la IP, a propósito de la incertidumbre respecto de la empleabilidad que enfrentan los futuros profesionales (Hirsch, 2013). En el caso de la psicología, es probable que quienes se forman profesionalmente como psicólogos, en la medida que avanzan en la carrera deban lidiar con estas preocupaciones. Además del cuestionamiento que ha recibido la disciplina como ciencia, y la dificultad de la profesión para posicionarse en un rol social más coherente en el abordaje del comportamiento humano (Covarrubias, 2013). 
De esta manera, la formación profesional de psicólogos desde un enfoque por competencias debe considerar que, para el desarrollo de la IP se requiere de la internalización de los estándares profesionales mediante variados métodos formativos. Por ejemplo, mediante una mayor coherencia entre la teoría y la práctica; entre el conocimiento científico y profesional; el subjetivo y formal.

Para Rønnestad y Skovholt (2003) la construcción de la IP se da de manera gradual a través de la formación universitaria, en donde los formadores deben considerar el conocimiento, crecimiento personal, experiencia, percepciones sobre la profesión, experiencias de logro, costos y relaciones interpersonales del estudiante.

El tercer objetivo de este trabajo fue proponer un modelo que explique la IP de estudiantes de psicología, en función de las variables estudiadas. Se encontró que las variables autoeficacia académica, autoeficacia profesional, créditos cursados, nivel de dificultad percibida ante la carrera e identidad estudiantil universitaria son predictoras de la IP. Lo que se graficó en un modelo que explica el $24 \%$ de la varianza de la IP. Aunque el tamaño del efecto es bajo $\left(R^{2}=0.24\right)$, aporta con evidencia empírica a los modelos ya propuestos. Se observa que la autoeficacia académica tiene un efecto directo y positivo con la IP, y que la autoeficacia profesional tiene un efecto positivo e indirecto sobre esta variable. Algunos autores (Camposeco, 2012) han relacionado la IP con la resolución adecuada de las tareas que enfrentan los estudiantes en sus procesos formativos, y la autoeficacia que desarrollan a partir de éstas. Así, la autoeficacia se propone como una variable predictiva de la IP (Canrinus, Helms-Lorenz, Beijaard, Buitink, \& Hofman, 2012; Cara \& Riveros, 2016; Flowers \& Banda, 2016).

También hemos encontrado que la identidad estudiantil universitaria es una variable predictora y mediadora entre la autoeficacia académica y la IP. Esto parece apoyar lo planteado por Lefroy et al. (2017), porque la autoeficacia ha demostrado incidir en la transición de una identidad estudiantil hacia una profesional. En ese proceso, la socialización con los pares y profesores promueven el desarrollo de la identidad profesional (Covarrubias, 2013), siempre que el estudiante logre identificarse con estos grupos (González, 2010).

Los créditos cursados y la dificultad percibida en la carrera también median como variables entre la autoeficacia académica y la IP. En el modelo presentado, los créditos cursados se han propuesto como una variable de progresión en la carrera de psicología, y se relacionan negativamente tanto con autoeficacia académica como con la identidad estudiantil universitaria; y esta última, con la IP. Respecto de esto, algunos autores (Covarrubias, 2013) han advertido de lo complejo que hoy por hoy es para los estudiantes de educación superior desarrollar una IP, y sobre todo en la carrera de psicología. En donde una mayor progresión en la carrera, podría conllevar a una mayor confusión por el cuestionamiento histórico de la psicología como ciencia; y lo difuso del rol profesional que, incluso podría llegar a cuestionar su identidad como estudiante universitario. Parecer ser que este problema emerge actualmente y que, en décadas pasadas la formación de la IP implicaba una menor complejidad; y efectivamente se lograba desarrollar en la media que los alumnos de psicología avanzaban en su carrera (Rønnestad \& Skovholt, 2003). 
Por otro lado, se observa un efecto mediador de la variable dificultad percibida en la carrera, en la incidencia de la autoeficacia académica sobre la IP. Así, un aumento de la autoeficacia académica podría implicar una menor percepción de dificultad en la carrera profesional, y esto último disminuir el desarrollo de la IP. Este efecto mediador puede comprenderse desde lo propuesto por Oyserman (2015), esto es que, las situaciones contextuales reportan información de la congruencia, importancia y dificultad de una tarea para la IP. Tareas percibidas demasiado fáciles o interpretadas como imposibles de realizar representan un sinsentido para la IP. Se requiere de una tarea congruente y con un grado de dificultad apropiado para que sea considerada importante y significativa para la IP.

Finalmente, es necesario plantear algunas limitaciones del estudio. En primer lugar, es necesario considerar que la confiabilidad obtenida en la escala de IP fue relativamente aceptable, por lo que los resultados de este estudio deben ser considerados dentro de este contexto. También alerta de la necesidad de que futuras investigaciones aporten con nuevas formas de medir este constructo. En segundo lugar, se debe asumir que el modelo presentado constituye solo una propuesta que emerge desde la literatura y se avala en los datos. Es necesario replicarlo en otros estudios, con muestras más amplias que posibiliten probar el poder explicativo y la invariabilidad con otros grupos, de acuerdo con el género, la tipificación de estudiante tradicional y no tradicional e incluso el tipo de institución de educación superior. No obstante, representa un primer paso para aproximarse a la comprensión de la formación de la IP en estudiantes de psicología.

\section{Referencias}

Adams, K., Hean, S., Sturgis, P., \& Clark, J. (2006). Investigating the factors influencing professional identity of first-year health and social care students. Learning in Health and Social Care, 5(2), 55-68.

Alegre, A. (2014). Autoeficacia académica, autorregulación del aprendizaje y rendimiento académico en estudiantes universitarios iniciales. Propósitos y Representaciones, 2(1), 79-120. doi: 10.20511/pyr2014.v2n1.54

Arias, M. \& Pineda, S. (2011). Conocimientos que tienen los estudiantes de una universidad pública de Manizales sobre el Papillomavirus humano. Hacia la Promoción de la Salud, 16(1), 110-123.

Arteaga, E., Joya, M., \& Bastidas, G. (2014). Identidad estudiantil universitaria en la Escuela de Medicina, Sede Carabobo, Universidad de Carabobo, Venezuela. Rev Educ Cienc Salud, 11(1), 18-25.

Bandura, A. (2006). Guide for constructing self-efficacy scales. In F. Pajares y T. Urdan (Eds.), Self-Efficacy Belief of Adolescents (307-337). Greenwich: Information Age Publishing.

Barahona, P. (2014). Factores determinantes del rendimiento académico de los estudiantes de la Universidad de Atacama. Estudios Pedagógicos, 40(1), 25-39.

Barbour, J. \& Lammers, J. (2015). Measuring profesional identity: a review of the literatura and a multilevel confirmatory factor analysis of professional 
identity constructs. Journal of professions and Organization, 2(1), 38-60. doi: 10.1093/jpo/jou009

Bentler, P. (1995). Structural equations program manual. Encino, CA: Multivariate Software, Inc.

Blanco, H., Ornelas, M., Aguirre, J., \& Guedea, J. (2012). Autoeficacia percibida en conductas académicas. Diferencias entre hombres y mujeres. Revista Mexicana de Investigación Educativa, 17(53), 557-571.

Briggs, A. (2007). Exploring professional identities: middle leadership in further education colleges. School Leadership and management, 27(5), 471-485.

Byrne, B. (2001). Structural equation modeling with AMOS: Basic concepts, applications, and programming. Mahwah, NJ: Lawrence Erlbaum.

Cabral, M., Villanueva, E., Estrada, G., González, S., Juárez, C., Hernández, ... Nacar, V. (2006). Identidad estudiantil universitaria en estudiantes de licenciatura. Revista Electrónica Psicología Cientifica. Recuperado de http:// www.psicologiacientifica.com/identidad-estudiates-universitarios.

Camposeco, F. (2012). La autoeficacia como variable en la motivación intrínseca y extrínseca en matemáticas a través de un criterio étnico (Tesis Doctoral). Universidad Complutense de Madrid, Madrid.

Canrinus, E., Helms-Lorenz, M., Beijaard, D., Buitink, J., \& Hofman, A. (2012). Self-efficacy, job satisfaction, motivation and commitment: exploring the relationships between indicators of teachers' professional identity. European Journal of Psychology of Education, 27, 115-132. doi: 10.1007/ s10212-011-0069-2

Cara, M. \& Riveros, R. (2016). Autoeficacia y transformación dinámica del aprendizaje en la práctica docente inicial. Perspectiva Educacional. Formación de Profesores, 55(1), 3-19.

Covarrubias, P. (2013). Imagen social e identidad profesional de la psicología desde la perspectiva de sus estudiantes. Revista Iberoamericana de Educación Superior, 4(10), 113-133.

Cuadra, D., Castro, P., \& Juliá, M. T. (2018). Tres saberes en la formación profesional por competencias: integración de teorías subjetivas, profesionales y científicas. Formación Universitaria, 11(5), 19-30. doi: 10.4067/S0718-50062018000500019

Díaz, C. (2016). Métodos de investigación en educación. Universidad Católica del Perú: Perú.

Donoso, S. \& Corvalán, O. (2012). Formación técnica y aseguramiento de la calidad: enfoque de desarrollo de competencias. Cadernos de Pequisa, 42(1), 612-639.

Egan, S. B. (2004). Role strain in female students in graduate social work education: Culturally competent institutional responses (Doctoral dissertation). Fordham 
University. Recuperado de http://proquest.umi.com/pqdweb?did=7658 $13901 \&$ sid $=1 \& \mathrm{Fmt}=2 \&$ clientId $=1509 \& \mathrm{RQT}=309 \& \mathrm{VName}=\mathrm{PQD}$.

Elman, N., Illfelder, J., \& Robiner, W. (2005). Professional develpment: training for professionalism as a foundation for competence practice in psychology. Professional Psychology: Research and Práctice, 36(4), 367-375.

Flowers, A. \& Banda, R. (2016). Cultivating science identity through sources of self-efficacy. Journal for Multicultural Education, 10(3), 405-417. doi: 10.1108/ JME-01-2016-0014

Giddens, A. (2010). Sociología. Madrid: Alianza Editorial.

González, J. (2010). Biografía, identidad y aprendizaje en estudiantes universitarios no tradicionales. Estudio de caso de una mujer trabajadora. Profesorado. Revista de Curriculum y Formación del Profesorado, 14(3), 131-147.

Gorman, E. \& Sandefur, R. (2011). “Golden age,” Quiescence, and revival: How the Sociology of Professions Became the Study of Knowledge-Based Work. Work and Occupations, 38(3), 275-302. doi: $10.1177 / 0730888411417565$

Hirsch, A. (2013). Elementos teóricos y empíricos acerca de la identidad profesional en el ámbito universitario. Perfiles Educativos, 35(140), 63-81.

Hoyert, M. S. \& D. O'Dell, C. (2009). Goal orientation and academic failure in traditional and nontraditional aged college students. College Student Journal, 43(4), 1052-1061.

Kaya, H., Işik, B., Şenyuva, E., \& Kaya, N. (2017). Personal and professional values held by baccalaureate nursing students. Nurs Ethics, 24(6), 716-731. doi: $10.1177 / 0969733015624488$

Lefroy, J., Yardley, S., Kinston, R., Gay, S., McBain, S., \& McKinley, R. (2017). Qualitative research using realist evaluation to explain preparedness for doctors' memorable 'firsts'. Medical Education, 51(10), 1037-1048. doi: $10.1111 /$ medu. 13370

Linnenbrink, E. A. \& Pintrich, P. R. (2003). The role of self-efficacy beliefs in student engagement and learning in the classroom. Reading and Writing Quarterly: Overcoming Learning Difficulties, 19(2), 119-137.

Maffei, L., Sponton, C., Sponton, M., Castellano, E., \& Medrano, L. (2012). Adaptación del Cuestionario de Autoeficacia Profesional (AU-10) a la población de trabajadores cordobeses. Pensamiento Psicológico, 10(1), 51-62.

Martínez-Otero, V. (2014). Ansiedad en estudiantes universitarios: estudio de una muestra de alumnos de la Facultad de Educación. Revista de la Facultad de Educación de Albacete, (29-2), 63-78. Recuperado de http://www.revista. uclm.es/index.php/ensayos 
Meschman, C., Erausquin, C., \& García, L. (2014). Huellas, herencias y tramas: construyendo la identidad profesional del Profesor de Psicología. Anuarios de Investigación, 21(1), 105-116.

Montes, I. \& Lerner, J. (2011). Rendimiento académico de los estudiantes de pregrado de la Universidad EAFIT. Colombia: Universidad EAFIT.

Negrillo, C. (2015). Análisis de la identidad profesional y la autodiscrepancia en estudiantes de enfermería (Tesis Doctoral). Universidad de Sevilla, Sevilla.

Olukemi, A. \& Awotedu, F. (2014). Challenges faced by married university undergraduate female students in Ogun State, Nigeria. Procedia-Social and Behavioral Sciences, 112, 102-113.

Oyserman, D. (2015). Identity-based motivation. In R. Scott and S. Kosslyn (Eds.), Emerging Trends in the Behavioral and Social Sciences, Hoboken, NJ: John Wiley and Sons. Published. doi: 10.1002/9781118900772.etrds0171

Penrose, A., Perry, C., \& Ball, I. (2007). Emotional intelligence and teacher selfefficacy: the contribution of teacher status and length of experience. Issues in Educational Research, 17, 107-126

Pérez, E., Medrano, L., \& Sánchez, R. (2013). El Path Analysis: conceptos básicos y ejemplos de aplicación. Revista Argentina de Ciencias del Comportamiento, 5(1), 52-66. Recuperado de www.psyche.unc.edu.ar/racc

Rønnestad, M. \& Skovholt, T. M. (2003). The journey of the counselor and therapist: Research indings and perspectives on professional development. Journal of Career Developmenter, 30(1), 5-44.

Ruvalcaba, J., Uribe, A., \& Gutiérrez, R. (2011). Identidad e identidad profesional: acercamiento conceptual e investigación contemporánea. Revista CES Psicología, 4(2), 82-102. Recuperado de https:/ / dialnet.unirioja.es/descarga/ articulo/3817911.pdf

Salanova, M., Bresó, E., \& Schaufeli, W. B. (2005). Hacia un modelo de las creencias de eficacia en el estudio del burnout y del engagement. Ansiedady Estrés, 11(2-3), 215-231.

Sánchez, A. \& Andreau, M. (2017). Los estudiantes universitarios no tradicionales y el abandono de los estudios. Estudios sobre Educación, 32, 27-48. doi: 10.15581/004.32.27-48

Scott, L. M., \& Lewis, C. W. (2012). Nontraditional college students: Assumptions, perceptions, and directions for a meaningful academic experience. International Journal of Interdisciplinary Social Sciences, 6(4), 1-10.

Sweitzer, V. (2008). Networking to develop a professional identity: a look al the first-semester experience of doctoral students in business. New Directions for teaching and learning, 113, 43-56.

Taylor, J. \& House, B. (2010). An exploration of identity, motivations and concerns of non-traditional students at different stages of higher education. Psychology Teaching Review,16(1), 46-57. 
Tilley, B. (2014). What Makes a Student Non-traditional? A comparison of students over and under age 25 in online, accelerated psychology courses. Psychology Learning and Teaching, 13(2), 95-106.

Torre Puente, J. (2006). La autoeficacia, la autorregulación y los enfoques de aprendizaje en estudiantes universitarios (Tesis doctoral). Madrid, Universidad Pontificia Comillas.

Trede, F. (2012). Role of work-integrated learning in developing professionalism and professional identity. Asia-Pacific Journal of Cooperative Education, 13(3), 159-167.

Velthuis, C., Fisser, P. \& Pieters, J. (2014). Teacher training and pre-service primary teachers' self-efficacy for science teaching. Journal of Science Teacher Education, 25, 445-464. doi:10.1007/s10972-013-9363-y

Zinkiewicz, L. \& Trapp, A. (2004). Widening and increasing participation. Challenges and opportunities for psychology departaments. Toronto: University of York. Recuperado de www.psychology.heacademy.ac.uk/docs/pdf/p20040422_widen_partic.pdf 